\title{
Borderline Ovarian Tumor with Severe Dyspnea in a 79 Year Old Patient
}

\section{Dominguez Gonzalez Manuel, Essarroukh Zaitouni Imane, Carrasco Trigueros María Amparo, Muñoz González María Dolores and Blanes Martínez Eugenio Jose}

Department of Obstetrics and Gynecology, Hospital La Linea, La Linea de la Concepcion, Cadiz, Spain

Corresponding author: Dominguez Gonzalez Manuel, Doctor of Obstetrics and Gynecology, Department of Gynecology and obstetrics, Hospital La Linea, Avenida Menéndez Pelayo, 103 s/n, La Linea de la Concepción, Cadiz, Spain, Tel: +34-956-026-500; E-mail: manueldominguezg@hotmail.com

Received date: July 19, 2016; Accepted date: January 10, 2017; Published date: January 12, 2017

Citation: Domínguez González M, Essarroukh Zaitouni I, Carrasco Trigueros MA, et al. Borderline Ovarian Tumor with Severe Dyspnea in a 79 Year Old Patient. Gynecol Obstet Case Rep. 2017, 3:1.

\section{Abstract}

Borderline ovarian tumors (also called tumors of malignancy potential) are heterogeneous team of lesions histologically defined by atypical epithelial proliferation without stromal invasion.

The majority of those tumors use to have a good forecast, what is not so habitual is that if the tumor has a big size in on elderly patient with basic cardiopulmonary pathology it can be a urgent situation that endangers the life of the patient.

We present a clinical case of a pluripathology 79 years old female who comes for consultation with tachycardiatachypnea, incapacity for supine decubitus and dyspnea on minimal effort. A large ovarian-dependent abdominal tumor is diagnosed. Borderline left ovary tumor of $42 \times 36$ $\times 7 \mathrm{~cm}$ is removed. Good postoperative evolution. Disappearance of all respiratory and digestive symptoms after surgery. Evolution after one year remained asymptomatic and free of disease.

Keywords: Borderline; Ovarian tumor; Tumors of low malignant potential; Dyspnea

\section{Introduction}

Borderline ovarian tumors (also called tumors of low malignant potential) are a heterogeneous group of lesions histologically defined by atypical epithelial proliferation without stromal invasion [1]. This group of neoplasms exhibits an intermediate behaviour between benign cystadenomas and carcinomas. Borderline tumors represent $14 \%$ to $15 \%$ of all primary ovarian neoplasms [2].

Most of these tumors usually have a good prognosis. What is not so common is that if the tumor is large in an elderly patient with underlying cardiopulmonary disease may be a serious situation due to secondary respiratory failure. We present a case of a giant ovarian borderline tumor in a 79 year old patient in whom poor general condition, cardiopulmonary disease and respiratory insufficiency make a benign tumor an emergency that compromises the patient's life.

\section{Clinical Case}

79 years old female patient who consults for tachycardiatachypnea and incapacity for supine decubitus due to severe dyspnea. Dyspnea at minimal effort with absence of previous pulmonary pathology. Refers loss of $30 \mathrm{~kg}$ of weight without reduction of the abdominal diameter. In addition to epigastralgia and digestive discomfort, initially studied by internal medicine, which refer to gynecologist for treatment of large abdominal mass of probable adnexal origin.

Regarding personal history, the patient has no known drug allergies with no toxic habit, hypertension, obesity, hyperlipidemia, hyperuricemia, permanent atrial fibrillation (in treatment with dicumarinics), hypertensive heart disease, mild-moderate mitral insufficiency with moderate pulmonary hypertension, no hiatus hernia or peptic ulcus, spondylarthrosis and gonarthrosis. Where her surgical history includes appendectomy and as obstetric history 2 deliveries and 2 cesareans with menopause at age of 51 .

Exploration includes poor general condition with dyspnea at rest, severe obesity, much distended abdomen. A large tumor that reaches the umbilical level is felt, which seems mobile. Atrophic external genitals, vagina and cervix. Bimanual palpation: Tumor lodged in the pelvis and reaches the umbilical level.

Transvaginal echo demonstrated a small-sized uterus with calcifications, linear endometrium, Douglas occupied by a large solid-cystic tumor that is impossible to measure in its entirety by vaginal or abdominal echo (about $30 \mathrm{~cm}$ approximately) with septa, papillae and negative Doppler, where they do not look attached and with no free liquid.

Computed Tomography Scanner revealed a mass of cystic type with septa of $23 \times 31 \mathrm{~cm}$ that displaces viscera, with no free fluid or lymphadenopathy. 
Blood analysis figured with CA125: $58.4 \mathrm{mlU} / \mathrm{ml}$ with all other markers within normal limits worsening of the general state and dyspnea so that surgical treatment is decided.

Exploratory laparotomy, left annexectomy, total hysterectomy, contralateral annexectomy, peritoneal lavage and inframesocolic omentectomy was performed. A $25.4 \mathrm{~kg}$ piece with $42 \times 36 \times 7 \mathrm{~cm}$ of left ovarian origin was retrieved, translucent and whitish smooth capsule with cystic cavities of serous content. No signs of infiltration of neighbouring organs, lymph node involvement or free fluid. Rest of cavity without pathological findings. Significant improvement of the respiratory symptoms was annotated after resection (Figures 1 and 2).

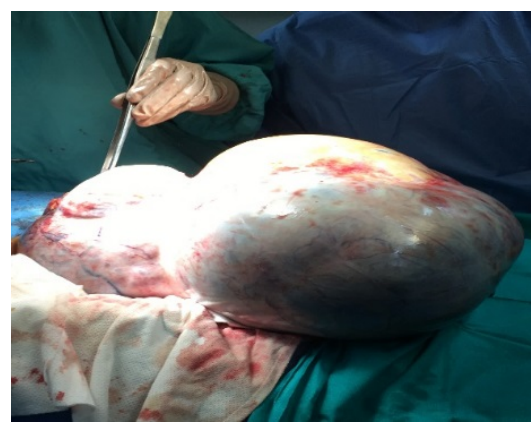

Figure 1 Tumor during surgical intervention.

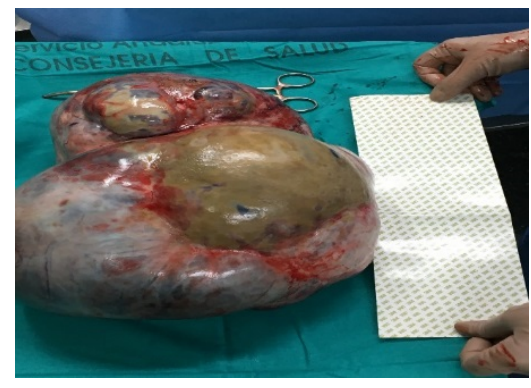

Figure 2 Tumor finally excised.

Postoperative evolution was favorable. All respiratory and digestive symptoms disappeared. The patient was discharged 7 days after the procedure.

Definitive anatomopathological result revealed atypical proliferative serous tumor (border line) of left ovary. Uterus, right annex and omentum without pathological findings. Negative peritoneal lavage for malignant cells: Stage I.

No additional treatment was given. After 1 year of followup, the patient continues asymptomatic with good quality of life. Magnetic resonance scanner of control was performed with no signs of relapse of the disease.

\section{Discussion}

Borderline tumors have a wide histological variety, as in ovarian epithelial carcinoma $[3,4]$. Most cases are serous or mucinous. On rare occasions we encounter endometrioid, clear cell, or transitional cell tumors also called "Brenner's Tumors".

The estimated incidence of borderline ovarian tumors varies from 1.8 to 5.5 per 100,000 women/year. Some data suggest that an increase in incidence is occurring [5].

The clinical presentation of borderline tumors is the same as for other adnexal masses. Some patients are asymptomatic at the time of diagnosis (approximately 14\%) [6]. In asymptomatic patients, can be detected by pelvic examination or imaging tests performed by another indication.

Pelvic or abdominal pain, pressure or dyspareunia are typical symptoms. Patients with borderline tumors are unlikely to present with ascites, intestinal obstruction, pleural effusion or thromboembolism. These symptoms and signs are more common in ovarian carcinoma. The clinical symptoms of severe dyspnea, tachypnea, tachycardia in a borderline ovary tumor is infrequent and may be fatal in an elderly patient with associated cardiac pathology if not treated promptly due to the compressive clinic as in our case. The diagnostic evaluation of a borderline ovarian tumor is the same as for ovarian carcinoma, except that the determination of serum CA 125 does not appear to be a useful test. There are no ultrasound features that differentiate a borderline ovarian tumor from benign or malignant ovarian neoplasia. The ultrasound aspect of borderline tumors ranges from unilocular cysts to complex masses with solid and cystic components. The presence of papillae is common $[7,8]$.

The particularity of our case is the abrupt worsening due to advanced age and the cardiac pathology that requires an urgent surgical treatment. The improvement of all the patient's constants was immediate after mass extirpation.

The diagnosis of the borderline tumor is histological after surgical removal of the ovary. Peritoneal dissemination is less frequent and in these cases the diagnosis can also be made by biopsies of the peritoneum. Most borderline tumors are serous. Approximately $75 \%$ of tumors are diagnosed in stage I. They can be bilateral in $25 \%$ to $50 \%$ of cases. The second most frequent histological type is the mucinous one [9].

The staging of borderline ovarian tumors is the same as for other invasive ovarian carcinomas of the fallopian tube or peritoneum. The complete staging procedure for ovarian cancer includes: total abdominal hysterectomy and bilateral salpingo-oophorectomy with peritoneal lavage, diaphragmatic dome cytology, omentectomy and resection of clearly visible metastases. In our case, there was no evidence of metastasis or signs of local tumor infiltration. Neither was lymphadenectomy performed (Lymphadenectomy is not recommended in this type of tumor) nor was chemotherapy administered. Adjuvant treatment with chemotherapy in borderline ovarian tumors is not usually performed. Chemotherapy does not provide benefit in the initial state in which the tumor has been completely removed [10]. The advanced stage is the main risk factor associated with recurrence, in addition to the histological characteristics of the tumor. The survival rate of 5 to 10 years in stage I is very high 
(97\% to 99\%) [11]. The risk of malignancy of this type of tumor is not clear.

\section{Conclusion}

The prognosis of borderline ovarian tumors depends on the stage and the histological features of the tumor, but is generally good. If the tumor is large in an elderly patient, it can cause severe respiratory symptoms due to compression (mass effect) and endanger the patient's life. It is necessary a multidisciplinary approach and an early surgical treatment to solve it.

\section{References}

1. Seidman JD, Kurman RJ (2003) Pathology of ovarian carcinoma. Hematol Oncol Clin North Am 17: 909-925.

2. Skírnisdóttir I, Garmo H, Wilander E, Holmberg L (2008) Borderline ovarian tumors in Sweden 1960-2005: Trends in incidence and age at diagnosis compared to ovarian cancer. Int J Cancer 123: 1897-1901.

3. Trimble CL, Trimble EL (2003) Ovarian tumors of low malignant potential. Oncology (Williston Park) 17: 1563-1567.

4. Harter P, Gershenson D, Lhomme C, Lecuru F, Ledermann J, et al. (2014) Gynecologic cancer intergroup (GCIG) consensus review for ovarian tumors of low malignant potential (borderline ovarian tumors). Int J Gynecol Cancer 24: S5-8.
5. Yahata T, Banzai C, Tanaka K, Niigata Gynecological Cancer Registry (2012) Histology-specific long-term trends in the incidence of ovarian cancer and borderline tumor in Japanese females: A population-based study from 1983 to 2007 in Niigata. J Obstet Gynaecol Res 38: 645-650.

6. Goff BA, Mandel LS, Melancon CH, Muntz HG (2004) Frequency of symptoms of ovarian cancer in women presenting to primary care clinics. JAMA 291: 2705-2712.

7. Valentin L, Ameye L, Testa A, Lécuru F, Bernard Jp et al. (2006) Ultrasound characteristics of different types of adnexal malignancies. Gynecol Oncol 102: 41-48.

8. Exacoustos C, Romanini ME, Rinaldo D, Amoroso C, Szabolcs B, et al. (2005) Preoperative sonographic features of borderline ovarian tumors. Ultrasound Obstet Gynecol 2005; 25: 50-59.

9. Lazarou A, Fotopoulou C, Coumbos A, Sehouli J, Vasiljeva J et al. (2014) Long-term follow-up of borderline ovarian tumors clinical outcome and prognostic factors. Anticancer Res 34: 6725-6730.

10. Tropé C, Kaern J, Vergote IB (1993) Are borderline tumors of the ovary overtreated both surgically and systemically? A review of four prospective randomized trials including 253 patients with borderline tumors. Gynecol Oncol 51: 236-243.

11. Trimble CL, Kosary C, Trimble EL (2002) Long-term survival and patterns of care in women with ovarian tumors of low malignant potential. Gynecol Oncol 86: 34-37. 